\title{
Crystallization of polyethylene: A molecular dynamics simulation study of the nucleation and growth mechanisms
}

\author{
Muhammad Anwar a, b, *, Tanja Schilling a \\ ${ }^{a}$ Physics and Materials Research Unit, Université du Luxembourg, L-1511 Luxembourg, Luxembourg \\ ${ }^{\mathrm{b}}$ Mechanical Engineering Department, Institute of Space Technology, Islamabad, Pakistan
}

\section{A R T I C L E I N F O}

\section{Article history:}

Received 15 May 2015

Received in revised form

24 July 2015

Accepted 21 August 2015

Available online 5 September 2015

\section{Keywords:}

Crystallization

Nucleation

Growth

Simulations

\begin{abstract}
A B S T R A C T
We have performed molecular dynamics simulations to study the mechanism of crystallization from an undercooled polyethylene (C500) melt. We observe that crystal nucleation is initiated by the alignment of chain segments, which is followed by straightening of the chains and densification. Growth procedes via alignment of segments, which are in the vicinity of the growth front, with the chains in the crystalline lamella. Once chains are attached, the lamella thickens by sliding of the segments along the long axis of the chain from the amorphous regions into the crystalline regions. We do not observe the formation of any folded precursors.
\end{abstract}

(c) 2015 Elsevier Ltd. All rights reserved.

\section{Introduction}

Semicrystalline polymers are widely used in industrial applications because of their mechanical properties. These properties depend on the morphology of the semicrystalline state, i.e. on the distribution of crystallites sizes and shapes, which is in turn caused by the crystallization process [1]. In the last seven decades a large amount of work has been done to understand the crystallization of polymers but still there are a number of question which are either unanswered or controversially answered [2,3]. A wide range of experimental techniques have been used to study the crystallization process of polymers under quiescent conditions [4-13] and under the influence of external fields [14-21] but the molecular level mechanisms of nucleation and growth have not yet been fully identified experimentally as they are difficult to resolve both, in space and time.

From the point of view of theory it is challenging to model the crystallization process, because relaxation of the polymer melt occurs on several time and length scales which makes it difficult to use quasi-equilibrium approaches. In such cases, computer simulations are powerful tools to unveil the molecular level

\footnotetext{
* Corresponding author. Mechanical Engineering Department, Institute of Space Technology, Islamabad, Pakistan

E-mail address: manwar18@gmail.com (M. Anwar).
}

mechanisms. In the last one and a half decades, many computer simulations studies of crystallization of polymers have been carried out [1,22-52]. In very few of these studies [31,32,36], the molecular mechanism of crystal growth has been addressed. However, nucleation in short chain alkanes has been simulated [22-27,29,30] and a scenario for the nucleation mechanism has been identified.

There are two recent simulation studies $[31,36]$ in which the growth mechanism has been addressed and the results do not agree with each other. As the systems were of different chemical composition (one was polyethylene the other PVA) agreement was not neccessarily expected, but one might have hoped to find a generally applicable picture. At the same time neither of the simulation results is consistent with the classical theories on polymer crystallization presented by Lauritzen and Hoffman (LH) [53] and Sadler and Gilmer (SD) [54].

In this article, we present a detailed analysis of the formation of crystal nuclei and revisit the molecular mechanism of crystal growth from the melt in polyethylene. We find yet another crystallization mechanism.

\section{Method}

\subsection{Model \& order parameters}

We have used a united atom model for polyethylene in which the chains consist of monomers that represent $\mathrm{CH}_{2}$ and $\mathrm{CH}_{3}$ groups 
$[41,55]$. The system parameters were the same as in ref. [56], apart from the Lennard-Jones cutoff radius, which we set to $r_{c}^{L J}=2.5 \sigma$, where $\sigma$ is the length scale given by the Lennard Jones interaction. We have used the ESPResSo package [57] to run the simulations and implemented the dihedral-cosine potential which was not present in the package by default.

In order to differentiate crystallites from the melt, we used several order parameters (we have previously applied the same approach to a melt of shorter chain alkanes $[30,58]$, thus we only briefly summarize it here. For details we refer the reader to ref. [30]). For the purpose of the analysis we split the polyethylene chains (C500) into segments of 15 monomers and computed the radius of gyration $R_{g}$ and the nematic order parameter $S_{2}$ of those segments that were involved in the formation of the critical nucleus.

In order to measure the local alignment of the chain segments, we computed the crystallinity order parameter. Monomers within a distance of $r_{c}=1.4 \sigma$ were considered as neighbours. We assigned a vector to every monomer $i$ pointing from monomer $i-1$ to $i+1$. Two neighbours $i$ and $j$ were considered as "aligned" if the assigned vectors to these monomers were almost parallel $\left(\theta_{i j} \leq 10^{\circ}\right)$. For a monomer to be considered "crystalline", it had to have at least 12 aligned neighbours. We obtained this value by sampling the probability distributions of the number of aligned neighbours in the bulk crystal and the bulk liquid.

\subsection{Simulation details}

We have performed molecular dynamics simulations of a system consisting of 200 chains of polyethylene (C500). The system was equilibrated at $500 \mathrm{~K}$ which is well above the melting temperature of polyethylene. We set the density to $0.89 \mathrm{~g} / \mathrm{cm}^{3}$ and performed all molecular dynamics simulations under constant volume and constant temperature conditions. We used an integration time step of $0.006 \tau$, where $\tau=\sqrt{\frac{k_{B} T}{m \sigma^{2}}}, m$ is the mass of a monomer, $k_{B}$ is Boltzmann's constant and T is the temperature in Kelvin.

After equilibrating the system at $500 \mathrm{~K}$, we quenched it to $280 \mathrm{~K}$ to observe the nucleation event and then heated it to $320 \mathrm{~K}$ to observe the growth process. We used the DPD thermostat [59] and the friction coefficient $\gamma$ for the thermostat was $1.0 \tau^{-1}$.

\section{Results and discussions}

\subsection{Nucleation mechanism}

To explore the nucleation mechanism, we identified the nucleation time $t_{0}$ (The time at which critical nucleus is formed.) and the monomers which participated in the formation of critical nucleus. We traced the structural and orientational properties of these monomers backward in time until they could not be distinguished from the melt monomers anymore. We analysed the formation of five nuclei in terms of the average radius of gyration $R_{g}$ of all chain segments that were part of the nucleus at $t_{0}$, the nematic order parameter $S_{2}$ of these chain segments, the local density in terms of the volume $V$ of the Voronoi cell associated to each monomer that is part of the nucleus and the crystallinity order parameter defined above.

In Fig. 1 we show the relative variations of these quantities with respect to the values they had at $-170 \Delta t$, where $\Delta t=100,000 \tau$. When we advance from the supercooled melt towards the formation of the critical nucleus at $t_{0}$, we observe first an increase in the global orientational order $S_{2}$, then an increase in the radius of gyration of the segments, and finally the local density increases and the crystal structure is formed. The mechanism we observed here is the same as

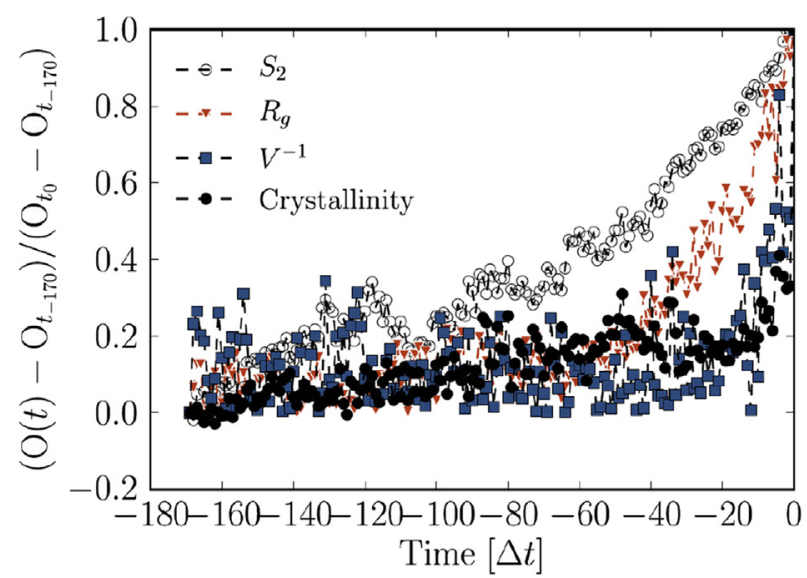

Fig. 1. Relative variation of several observables $(0)$ from the melt to the formation of a critical nucleus, computed for those monomers that are part of the nucleus at the nucleation time $t=t_{0}$ : orientational order $S_{2}$ (black, open circles), radius of gyration $R_{g}$ (red, triangles), the local density in terms of the inverse of the Voronoi cell volume $V$ (blue, squares) and the crystallinity order parameter (black, closed circles). The curves are averaged for the evolution of the five largest clusters progressing backward in time from the nucleation time $t=t_{0}$ in steps $\Delta t=100,000 \tau$ to $t=-170 \Delta t$. (For interpretation of the references to colour in this figure legend, the reader is referred to the web version of this article.)

the one we have found in short polymer chains [30,58]. We conclude that, at $280 \mathrm{~K}$ the undercooling is so strong that nucleation is a local event which does not depend on the chain length.

Snapshots of the critical nucleus are shown in Fig. 2. In the top view (Fig. 2 (b)) one sees that the stems are arranged hexagonally. In Fig. 2(c) we show the complete chain if more than one stems are formed by the chain, otherwise we only show the segment associated to these monomers. Several chains participate in the nucleation event with more than one stem.

\subsection{Growth mechanism}

To study the growth mechanism, we picked a configuration at

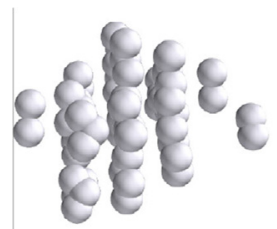

(a)

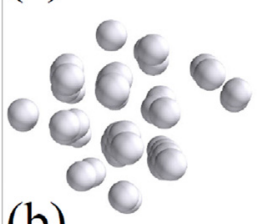

(b)

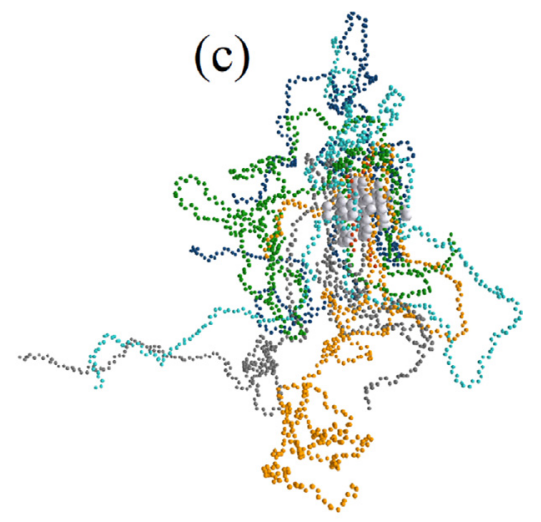

Fig. 2. Snapshots of the critical nucleus. (a): Only those monomers which are forming the critical nucleus at time $t_{0}$. (b): Top view of monomers which are forming the critical nucleus at time $t_{0}$. (c): Gray monomers: monomers that form the critical nucleus at $t_{0}$. Red monomers: segments of chains that participate with a single stem in the formation of the critical nucleus. Blue, green, cyan, battleship gray and orange monomers: chains which fold back and participate in the formation of the critical nucleus with more than one stem. For the case of folded chains we show complete chains instead of segments so that folds and tails can be identified. (For interpretation of the references to colour in this figure legend, the reader is referred to the web version of this article.) 

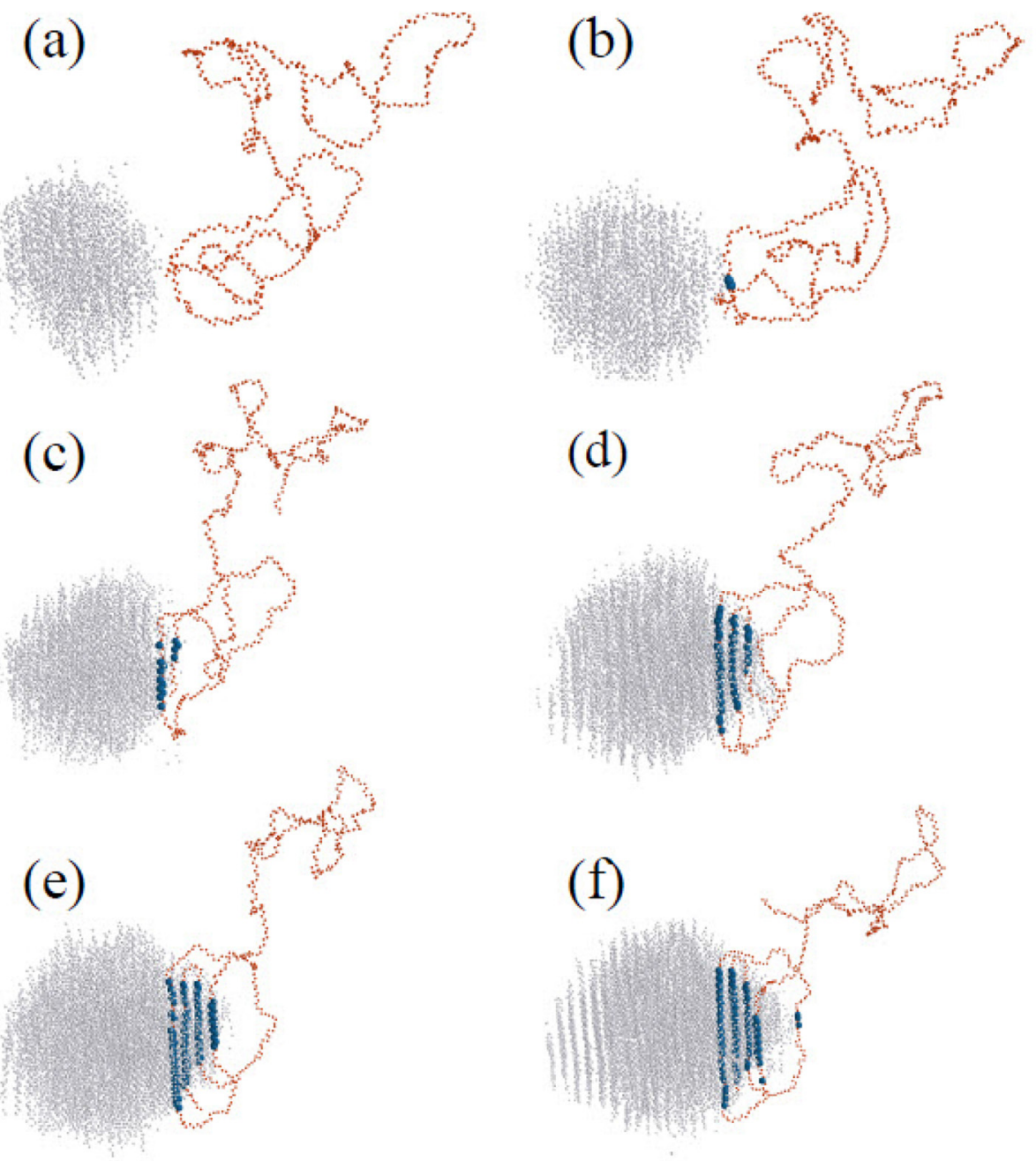

(g)

(h)
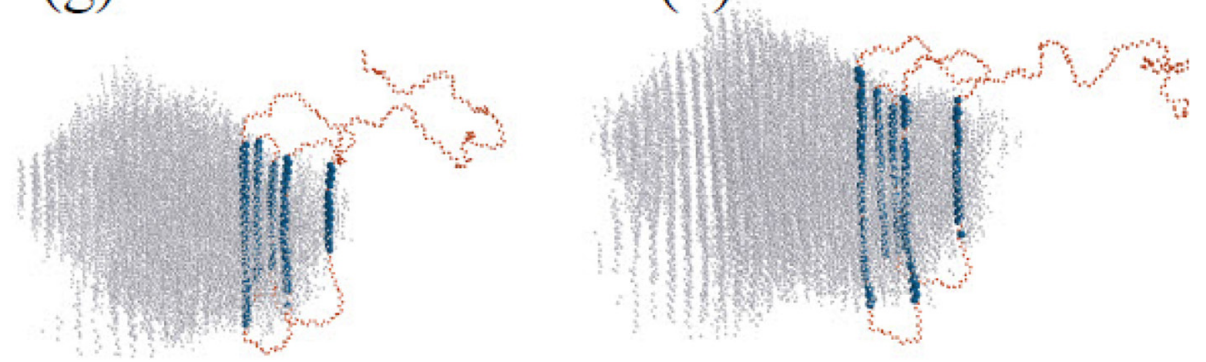

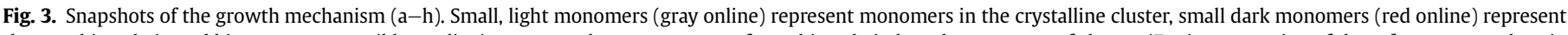

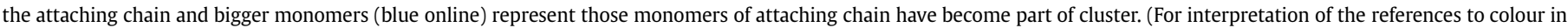
this figure legend, the reader is referred to the web version of this article.)

$280 \mathrm{~K}$ in which there was a crystalline cluster of 2047 monomers. We used this configuration as an initial configuration and ran simulations at $320 \mathrm{~K}$ so that we could see the growth of a single cluster in the system undisturbed by subsequent nucleation events. We show snapshots of the attachment of a single chain in Fig. 3. Light, small monomers (grey online) represent the monomers of the crystalline cluster, darker, small monomers (red online) represent the attaching chain and large monomers (blue online) represent those monomers of the attaching chain that have become part of the cluster. In Fig. 3(a), the chain is in the proximity of the cluster. In Fig. 3(b), a segment of this chain got aligned with the cluster and attached to the cluster. In Fig. 3(c), a new segment which is near to the cluster got attached to the cluster by getting aligned with the cluster and at the same time the already attached segment is getting straightened (thickening of lamella). In the same way the third, fourth and fifth segments are attaching on the cluster and the previously attached segments are getting thicker in Fig. 3(d)-(f) respectively. In Fig. 3(g-h), no new segment of this chain are attached but the lamella thickens.

In molecular dynamics simulations of poly(vinyl acohol) [36] it has been observed that the chains which are on the edge of the lamella form a number of short folded stems instantaneously which 
then act as precursors for further growth. Another simulation study on crystal growth in polyethylene [31] found that the segments of the chains which are on the edge of the growth front form a hairpin-like structure which is then attached on the growth front. In our simulation trajectories, we neither observe any folded precursors nor any hairpin structures before the attachment of the segments. We observe that once a segment of a chain is in the vicinity of the crystal cluster, it orients itself parallel to the stems in the cluster and attaches, then it straightens and the lamella thickens by reeling in of the chain segments from the amorphous regions. The difference in mechanism when compared to the observations by Yamamoto [31] might stem from the different polyethylene models used or from the fact that a perfectly crystalline substrate has been prepared in ref. [31], while we used a crystallite from a nucleation event.

Thickening of the lamella occurs through sliding of the chains along their axes as shown in Fig. 4(a-d). In this figure, gray monomers represent the complete chain, two monomers on the chain are highlighted (red online) to show the motion of the chain, and the dark monomer (blue online) mark the end of the chain. The crystalline stems gets thicker by pulling the amorphous segments of the chains through sliding along the long axes. In Fig. 4(e), we show the sliding velocity of the crystalline monomers along the axis of the stems. First, it increases slowly and then speeds up until

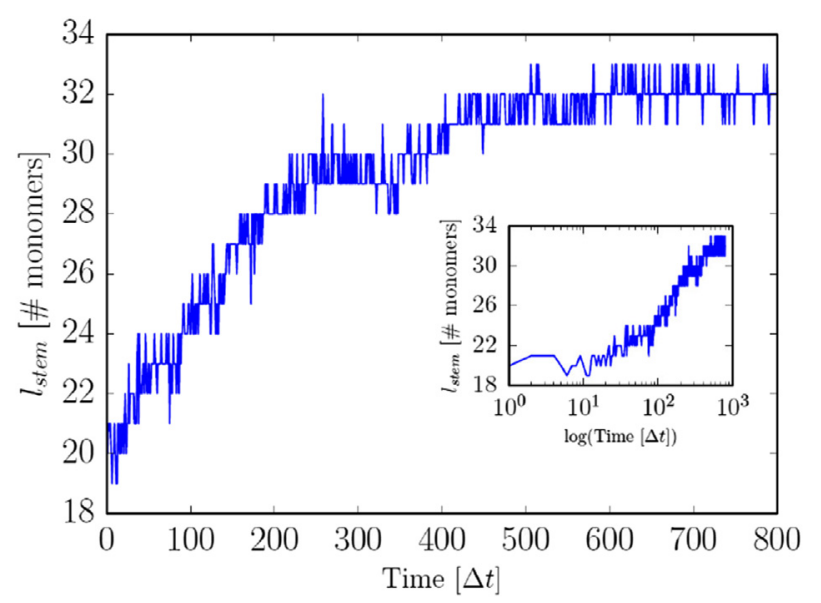

Fig. 5. Main: Average stem length as a function of time. Inset: Average stem length as a function of logarithm of time.

it saturates. The time-scale on which saturation is reached is deep in the logarithmic growth regime, which we introduce in the next paragraph.

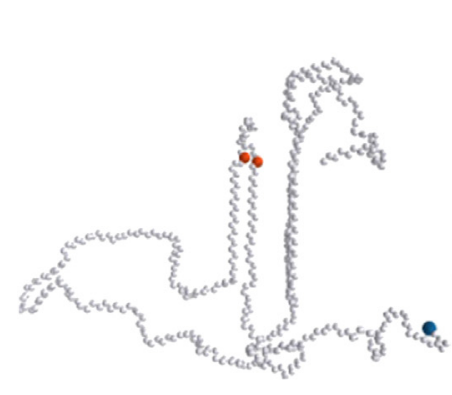

(a)

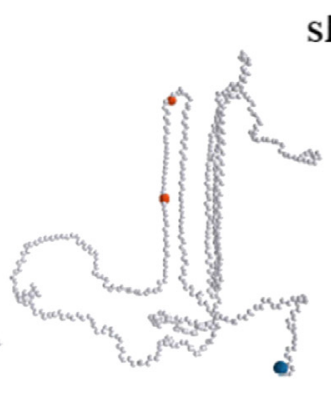

(b) sliding velocity

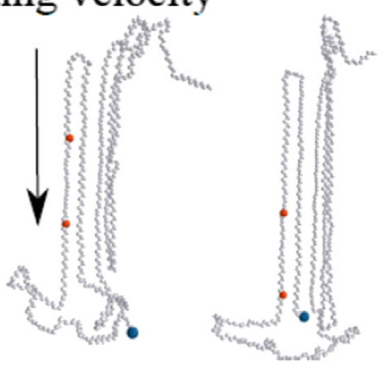

(c)

(d)

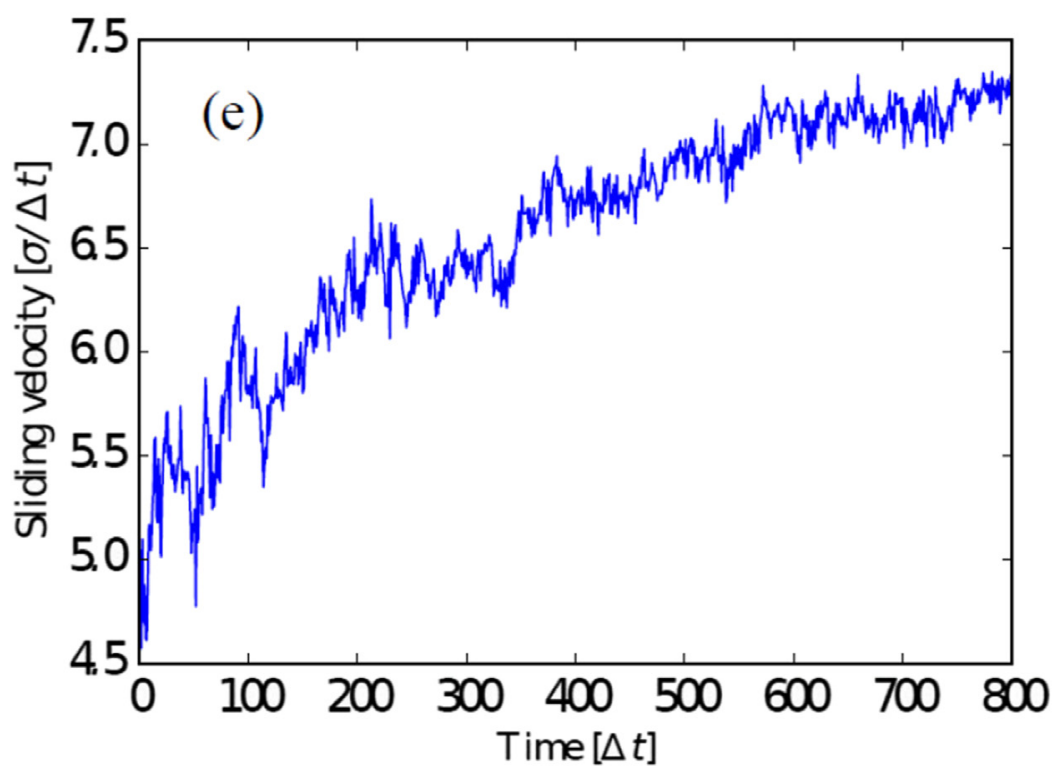

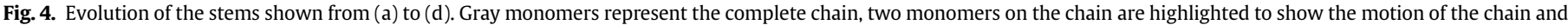

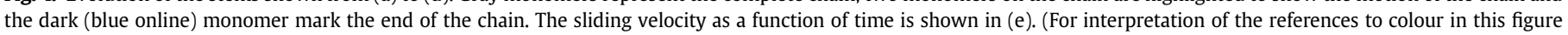
legend, the reader is referred to the web version of this article.) 
In Fig. 5, we show average stem length as a function of time. It increases linearly in time till $t=200 \Delta t$. Then there is a cross-over to a logarithmic growth law (See inset, in which we show the same quantity on semilogarithmic scale). Thickening behaviour of this type has already been observed by Luo and Sommer [36] who proposed a model in which the first regime is governed by the enthalpy cost of forming precursor stems, while the second regime is governed by the slipping of monomers over an energetic barrier (i.e. a hopping process along the chain).

In Fig. 6, we show the inter-stem distance in the lamella at time $t=800 \Delta t$, where $\Delta t=100000 \tau$ (for different times $t=0 \Delta t$ to $t=800 \Delta t$ we find the distributions to look the same, thus we only show one example). Adjacent folding is the dominant folding mechanism. (This observation is in agreement with ref. [31].) In the insets of Fig. 6 we plot the probability distribution of the stem length and provide information on the cluster size, number of stems, average stem length and number of chains participating in the lamella at that particular time step. In the inset, the probability distributions of the stem length show maximal values around the average stem length. This shape of the curve is due to the tapered structure of the lamella.

In Fig. 7, we show the distribution of the number of monomers in the folds (i.e. between attached stems) at time $t=800 \Delta t$, where $\Delta t=100000 \tau$. There are very few tight loops, and on average there are 39 monomers between the stems. This supports our previous observation that the growth mechanism does not proceed by formation of hairpin structures. We again checked these distributions at different timesteps ranging from $t=0 \Delta t$ to $t=800 \Delta t$ and we find them to be similar.

In Fig. 8, we show the growth rate as a function of degree of supercooling. To compute the growth rate at different temperatures, we picked a configuration with a crystalline cluster of size 4007 monomers and ran the simulations for $200 \Delta t$ timesteps. We find the familiar behaviour with an increase of the growth rate on decreasing temperature due to the increased thermodynamic driving force and then a decrease due to the slow-down of the dynamics.

\section{Conclusions}

We performed a molecular dynamics simulations to study the

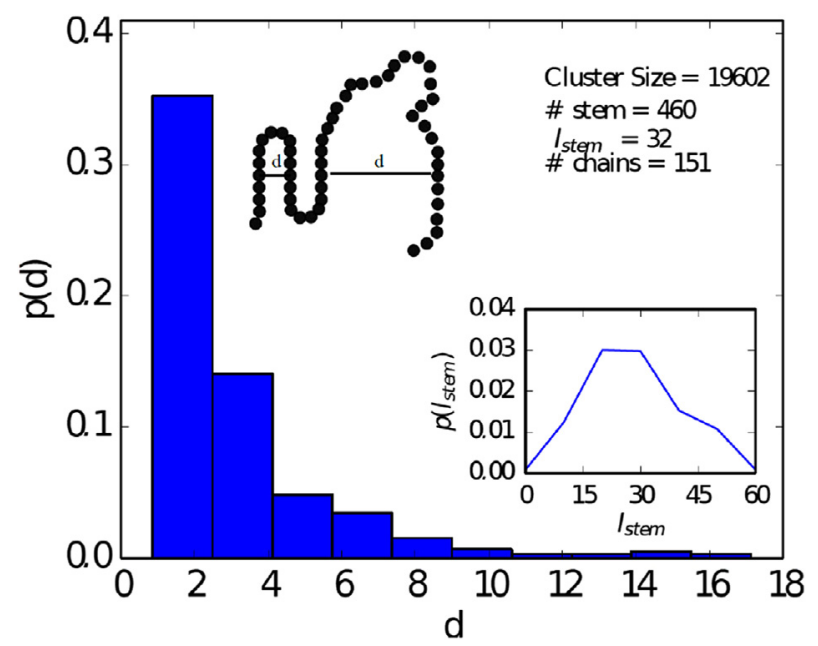

Fig. 6. Probability distribution of the inter-stem distance between the ends of the folds of the chains shown for time $t=800 \Delta t$. A sketch of the inter-stem distance in the folded crystal structure is also shown. In the inset, we draw the probability of the stem length in the system and we also provide information on the cluster size, number of stems, average stem length and number of chains participating in the lamella.

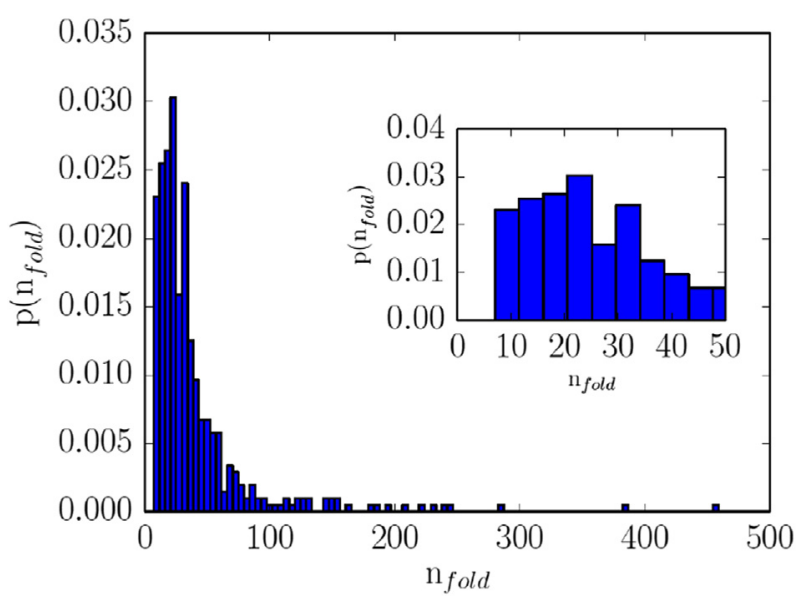

Fig. 7. Main: Probability distribution of the monomers in the fold (i.e. between stems). Inset: Probability distribution of the monomers in the fold for small fold lengths.

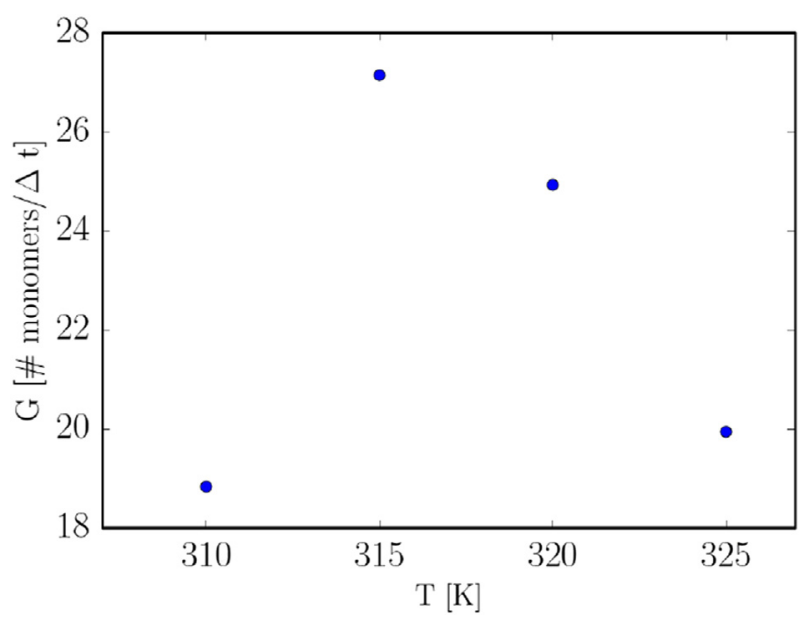

Fig. 8. Growth rate as a function of degree of supercooling.

microscopic mechanisms of nucleation and growth in a polyethylene melt. We observe that nucleation is initiated by the alignment of the chain segments which participate in the formation of critical nucleus, similar to the mechanism previously observed for shorter chains $[30,58]$. During growth, the segments of the chains which are close to the growth front align with the long axis of the stems to attach to the cluster and then thicken by sliding/ slipping from the amorphous regions into the crystalline regions. Thickening of the lamella procedes linearly in time at short times and logarithmically at longer times. In the attachment process, stems which are part of the same chain tend to be incorporated into the cluster closely to each other. However this does not imply that they form hairpins, as there are long liquid-like sections of the chain between the stems.

\section{Acknowledgements}

We thank Francesco Turci, Jens-Uwe Sommer, Roland Sanctuary, Jörg Baller and Carlo Di Giambattista for stimulating discussions. This project has been financially supported by the National Research Fund (FNR) (FNR/784560) within the CORE project Polyshear. Computer simulations presented in this paper were carried out using the HPC facility [60] of the University of Luxembourg. 


\section{References}

[1] A. Jabbarzadeh, R.I. Tanner, Crystallization of alkanes under quiescent and shearing conditions, J. Newt. Fluid Mech. 160 (1) (2009) 11-21.

[2] Günter Reiter, Gert R. Strobl, Progress in understanding of polymer crystallization, Lect. Notes Phys. 714 (2007). Springer-Verlag Berlin Heidelberg.

[3] Günter Reiter, Jens-Uwe Sommer, Polymer Crystallization: Obervations, Concepts and Interpretations, Springer, Berlin, Heidelberg, 2003.

[4] M. Imai, K. Kaji, T. Kanaya, Structural formation of poly(ethylene terephthalate) during the induction period of crystallization. 3. evolution of density fluctuations to lamellar crystal, Macromolecules 27 (24) (1994) 7103-7108.

[5] M. Imai, K. Kaji, T. Kanaya, Y. Sakai, Ordering process in the induction period of crystallization of poly(ethylene terephthalate), Phys. Rev. B 52 (1995) 12696-12704.

[6] T.A. Ezquerra, E. López-Cabarcos, B.S. Hsiao, F.J. Baltà-Calleja, Precursors of crystallization via density fluctuations in stiff-chain polymers, Phys. Rev. E 54 (Jul 1996) 989-992.

[7] A. Keller, M. Hikosaka, S. Rastogi, A. Toda, P.J. Barham, G. Goldbeck-Wood, An approach to the formation and growth of new phases with application to polymer crystallization: effect of finite size, metastability, and Ostwald's rule of stages, J. Mater. Sci. 29 (10) (1994) 2579-2604.

[8] M. Imai, K. Mori, T. Mizukami, K. Kaji, T. Kanaya, Structural formation of poly(ethylene terephthalate) during the induction period of crystallization:1. ordered structure appearing before crystal nucleation, Polymer 33 (1992) 4451.

[9] G. Strobl, From the melt via mesomorphic and granular crystalline layers to lamellar crystallites: a major route followed in polymer crystallization? Eur. Phys. J. E 3 (2) (2000) 165-183.

[10] G. Strobl, A thermodynamic multiphase scheme treating polymer crystallization and melting, Eur. Phys. J. E 18 (3) (2005) 295-309.

[11] G. Strobl, Crystallization and melting of bulk polymers: New observations, conclusions and a thermodynamic scheme, Prog. Polym. Sci. 31 (2006) 398-442.

[12] G. Strobl, T.Y. Cho, Growth kinetics of polymer crystals in bulk, Eur. Phys. J. E 23 (2007) 55.

[13] G. Strobl, Colloquium: laws controlling crystallization and melting in bulk polymers, Rev. Mod. Phys. 81 (2009) 1287-1300.

[14] R.H. Somani, L. Yang, I. Sics, B.S. Hsiao, N.V. Pogodina, H.H. Winter, P. Agarwal, H. Fruitwala, A. Tsou, Orientation-induced crystallization in isotactic polypropylene melt by shear deformation, Macromol. Symp. 185 (1) (2002) 105-117.

[15] Rajesh H. Somani, Ling Yang, Benjamin S. Hsiao, Precursors of primary nucleation induced by flow in isotactic polypropylene, Phys. A Stat. Mech. Appl. 304 (1-2) (2002) 145-157 (Scattering studies of mesoscopic scale structure and dynamics in soft matter).

[16] Ferass M. Abuzaina, Benjamin D. Fitz, Saša Andjelić, Dennis D. Jamiolkowski, Time resolved study of shear-induced crystallization of poly(p-dioxanone) polymers under low-shear, nucleation-enhancing shear conditions by small angle light scattering and optical microscopy, Polymer 43 (17) (2002) 4699-4708.

[17] D. Lellinger, G. Floudas, I. Alig, Shear induced crystallization in poly(€-caprolactone): effect of shear rate, Polymer 44 (19) (2003) 5759-5769.

[18] Salvatore Coppola, Luigi Balzano, Emilia Gioffredi, Pier Luca Maffettone, Nino Grizzuti, Effects of the degree of undercooling on flow induced crystallization in polymer melts, Polymer 45 (10) (2004) 3249-3256.

[19] Aadil Elmoumni, H. Henning Winter, Large strain requirements for shearinduced crystallization of isotactic polypropylene, Rheol. Acta 45 (6) (2006) 793-801.

[20] S. Acierno, N. Grizzuti, Flow-induced crystallization of polymer: theory and experiments, Int. J. Material Form. 1 (1) (2008) 583-586.

[21] Ri-Chao Zhang, Ai Lu, Zhong-Bin Xu, Effect of molecular weight on crystallization of semirigid poly(phenylene sulfide) under shear flow, J. Appl. Polym. Sci. 124 (2) (2012) 1562-1569.

[22] K. Esselink, P.A.J. Hilbers, B.W.H. van Beest, Molecular dynamics study of nucleation and melting of n-alkanes, J. Chem. Phys. 101 (10) (1994) 9033-9041.

[23] Hisao Takeuchi, Structure formation during the crystallization induction period of a short chain-molecule system: a molecular dynamics study, J. Chem. Phys. 109 (13) (1998) 5614-5621.

[24] Susumu Fujiwara, Tetsuya Sato, Molecular dynamics simulation of structural formation of short polymer chains, Phys. Rev. Lett. 80 (Feb 1998) 991-994.

[25] Susumu Fujiwara, Tetsuya Sato, Molecular dynamics simulation of structure formation of short chain molecules, J. Chem. Phys. 110 (19) (1999) 9757-9764.

[26] Peng Yi, Gregory C. Rutledge, Molecular simulation of crystal nucleation in noctane melts, J. Chem. Phys. 131 (2009) 1-12.

[27] Peng Yi, Gregory C. Rutledge, Molecular simulation of bundle-like crystal nucleation from n-eicosane melts, J. Chem. Phys. 135 (2011) 11.

[28] Peng Yi, C. Rebecca Locker, Gregory C. Rutledge, Molecular dynamics simulation of homogeneous crystal nucleation in polyethylene, Macromolecules 46 (11) (2013) 4723-4733.

[29] Hasan Zerze, Jeetain Mittal, Anthony J. McHugh, Ab initio crystallization of alkanes: Structure and kinetics of nuclei formation, Macromolecules 46 (22) (2013) 9151-9157.

[30] Muhammad Anwar, Francesco Turci, Tanja Schilling, Crystallization mechanism in melts of short n-alkane chains, J. Chem. Phys. 139 (21) (2013).
[31] Takashi Yamamoto, Molecular dynamics of polymer crystallization revisited: crystallization from the melt and the glass in longer polyethylene, J. Chem. Phys. 139 (5) (2013).

[32] Takashi Yamamoto, Molecular dynamics simulations of polymer crystallization in highly supercooled melt: Primary nucleation and cold crystallization, J. Chem. Phys. 133 (3) (2010).

[33] Takashi Yamamoto, Molecular dynamics simulations of steady-state crystal growth and homogeneous nucleation in polyethylene-like polymer, J. Chem. Phys. 129 (18) (2008)

[34] Takashi Yamamoto, Molecular dynamics modeling of polymer crystallization from the melt, Polymer 45 (4) (2004) 1357-1364 (Modeling of Chain Conformations and Spatial Configurations).

[35] Takashi Yamamoto, Molecular-dynamics simulation of polymer ordering. crystallization from vapor phase, J. Chem. Phys. 109 (11) (1998) 4638-4645.

[36] Chuanfu Luo, Jens-Uwe Sommer, Growth pathway and precursor states in single lamellar crystallization: Md simulations, Macromolecules 44 (6) (2011) 1523-1529.

[37] Chuanfu Luo, Jens-Uwe Sommer, Coexistence of melting and growth during heating of a semicrystalline polymer, Phys. Rev. Lett. 102 (Apr 2009) 147801

[38] P. Welch, M. Muthukumar, Molecular mechanisms of polymer crystallization from solution, Phys. Rev. Lett. 87 (Nov 2001) 218302.

[39] Hendrik Meyer, Florian Müller-Plathe, Formation of chain-folded structures in supercooled polymer melts, J. Chem. Phys. 115 (17) (2001) 7807-7810.

[40] Jens-Uwe Sommer, Günter Reiter, Polymer crystallization in quasi-two dimensions. ii. kinetic models and computer simulations, J. Chem. Phys. 112 (9) (2000) 4384-4393.

[41] N. Waheed, M.S. Lavine, G.C. Rutledge, Molecular simulation of crystal growth in n-eicosane, J. Chem. Phys. 116 (5) (2002) 2301-2309.

[42] M. Muthukumar, Modeling Polymer Crystallization, in: Advances in Polymer Science, vol 191, Springer Berlin Heidelberg, 2005, pp. 241-274.

[43] M. Muthukumar, Molecular modeling of nucleation in polymers, Philos. Trans. R. Soc. Lond. A Math. Phys. Eng. Sci. A361 (2003) 539-556.

[44] M. Muthukumar, Commentary on theories of polymer crystallization, Eur. Phys. J. E 3 (2) (2000) 199-202.

[45] Akira Koyama, Takashi Yamamoto, Koji Fukao, Yoshihisa Miyamoto, Molecular dynamics simulation of polymer crystallization from an oriented amorphous state, Phys. Rev. E 65 (050801) (May 2002).

[46] Akira Koyama, Takashi Yamamoto, Koji Fukao, Yoshihisa Miyamoto, Molecular dynamics studies on polymer crystallization from a stretched amorphous state, J. Macromol. Sci. Part B 42 (3-4) (2003) 821-831.

[47] Marc S. Lavine, Numan Waheed, Gregory C. Rutledge, Molecular dynamics simulation of orientation and crystallization of polyethylene during uniaxia extension, Polymer 44 (5) (2003) 1771-1779.

[48] Min Jae Ko, Numan Waheed, Marc S. Lavine, Gregory C. Rutledge, Charac terization of polyethylene crystallization from an oriented melt by molecular dynamics simulation, J. Chem. Phys. 121 (6) (2004) 2823-2832.

[49] T.C. Ionescu, C. Baig, B.J. Edwards, D.J. Keffer, A. Habenschuss, Structure formation under steady-state isothermal planar elongational flow of n-eicosane: a comparison between simulation and experiment, Phys. Rev. Lett. 96 (037802) (Jan 2006).

[50] I. Dukovski, M. Muthukumar, Langevin dynamics simulations of early stage shish-kebab crystallization of polymers in extensional flow, J. Chem. Phys. 118 (14) (2003) 6648-6655.

[51] Chunggi Baig, Brian J. Edwards, Atomistic simulation of crystallization of a polyethylene melt in steady uniaxial extension, J. Newt. Fluid Mech. 165 (17-18) (2010) 992-1004. Proceedings of the 5th International Workshop on Non-Equilibrium Thermodynamics 2009.

[52] C. Baig, B.J. Edwards, Atomistic simulation of flow-induced crystallization at constant temperature, Europhys. Lett. 89 (2010) 36003.

[53] G. Thomas Davis, D. Hoffman John, John I. Lauritzen Jr., Treatise on Solid State Chemistry, in: N.B. Hannay (Ed.), Crystalline and Noncrystalline Solids, Chapter the Rate of Crystallization of Linear Polymers with Chain Folding, vol. 3, Plenum Press, New York, 1976, pp. 497-614.

[54] D.M. Sadler, G.H. Gilmer, A model for chain folding in polymer crystals: rough growth faces are consistent with the observed growth rates, Polymer 25 (1984) 1446-1453.

[55] Wolfgang Paul, Do Y. Yoon, Grant D. Smith, An optimized united atom mode for simulations of polymethylene melts, J. Chem. Phys. 103 (4) (1995 $1702-1709$.

[56] N. Waheed, M.J. Ko, G.C. Rutledge, Molecular simulation of crystal growth in long alkanes, Polymer 46 (20) (2005) 8689-8702.

[57] H.J. Limbach, A. Arnold, B.A. Mann, C. Holm, ESPResSo-an extensible simulation package for research on soft matter systems, Comput. Phys. Commun. 174 (9) (2006) 704-727.

[58] Muhammad Anwar, Joshua T. Berryman, Tanja Schilling, Crystal nucleation mechanism in melts of short polymer chains under quiescent conditions and under shear flow, J. Chem. Phys. 141 (12) (2014).

[59] Thomas Soddemann, Burkhard Dünweg, Kurt Kremer, Dissipative particle dynamics: a useful thermostat for equilibrium and nonequilibrium molecular dynamics simulations, Phys. Rev. Lett. E 68 (Oct 2003) 046702.

[60] S. Varrette, P. Bouvry, H. Cartiaux, F. Georgatos, Management of an academic HPC cluster: the UL experience, in: Proc. of the 2014 Intl. Conf. on High Performance Computing \& Simulation (HPCS 2014), IEEE, Bologna, Italy, July 2014, pp. 959-967. 